\title{
Research on the Improvement of Employee's Innovation Performance Under Moral Leadership
}

\author{
Yuping Fang ${ }^{1}$ \\ ${ }^{1}$ Zhejiang Gongshang University School of Business Administration Hangzhou, China
}

\begin{abstract}
With the rapid development of technology and economy, more and more local Chinese enterprises are gradually growing and becoming prominent in all walks of life. However, the environment is complex and changeable, and various competitors and pressures make the importance of business management more prominent. One of the main purpose of business management is to improve enterprise performance so as to obtain more profits. Therefore, enterprise managers began to take a variety of measures in order to obtain higher performance. As an effective source to improve performance, innovation has become a goal that enterprise managers are chasing. The level of innovation performance has thus become one of the standards to measure the development of enterprises. Employees are the driving force of enterprise development, many a mickle makes a muckle, so in this process, their individual innovation performance is crucial, which is directly affected by the leadership style. Leadership has always been one of the hot topics in the field of business management and it has a direct impact on the effectiveness of business management. The main purpose of our study is to explore the underlying mechanisms that how moral leadership impacts employees' innovation performance by examining positive psychological safety's moderating role and voice behavior's mediating role of subordinates. We found that moral leadership is positively correlated with employee's innovation performance and this relationship is mediated by employees' voice behavior. Our results also demonstrate that employees' psychological safety moderates the positive relationship between moral leadership and voice behavior. Compared with a high level of psychological safety, this relationship is stronger when it is low.
\end{abstract}

\section{INTRODUCTION}

Under the current innovation-oriented social background, more and more enterprises begin to attach importance to the individual innovation performance of employees, and strive to find effective ways. Empirical research under the Chinese culture have demonstrated that leadership plays an important role in organizational processes because of its effects on employees' innovation performance12. It is worth exploring the specific type of leadership that can promote the innovative performance of employees. The concept of moral leadership comes from the paternalistic leadership which is a kind of indigenous leadership style in China and characterized by integrity and unselfish, in addition, it derives from China's Confucius values has been regarded as the strongest predictive ability on employees' job attitude and performance among the three paternalistic leadership styles. As an inconvenient leadership style, moral leadership has engaged the attention of organizational researchers all over the world in the past years. The relationship between moral leadership and followers' performance has been made clear in most of the study of moral leadership, less emphasized, however, is employees' behavioral mechanism underlying the relationship linking moral leadership to innovation performance.

In this study, we present that followers' proactive behavior, particularly voice behavior, acts as an momentous role in linking moral leadership with innovation performance. In view of the Chinese social and cultural backgrounds, we consider a proactive behavior mechanism as the process conveying the influence of moral leadership on followers' innovation performance. But the process is influenced by personal and environmental factors, so we also propose that the positive relationship linking moral leadership to voice behavior is moderated by psychological safety, which will contribute to improving employees' voice behavior and thus improve their innovation performance3. This view is also supported by complementary congruity theory, which supposes that leaders' abilities can fill the deficiencies, but required, component valued by followers.

We hold that moral leaders' abilities are conducive to complementing the required capabilities of employees for giving advice and suggestions actively. Broadly speaking, we could consider that moral leaders provide employees with complementary congruity, which has the impact on voice behavior. Whereas, the question to be answered is whether employees with different levels of psychological safety will be influenced to a greater or lesser extent by their moral leaders. But equally important is that 
psychological safety is such a belief that showing the dangerous behaviors, like voice behavior, will not get any stick. Whereas psychological safety as a moderator that may prompt employees' voice behaviors to further enhance their innovation performance has received few examined. Voice behavior can effectively promote employees' innovative performance, but employees tend to keep silent if they are worried that their opinions will bring embarrassment to themselves or other colleagues. What we are interested in is not only whether psychological safety moderates the moral leadership employees' voice behavior relationship but also the moderate effect between voice behavior and employees' innovation performance.

Based on Chinese culture, and social exchange theory and complementary congruity theory use for reference, the first aim of this study is to expand our knowledge of moral leadership by investigating how it promotes subordinates' voice behavior and in turn inspires their innovation performance in Mainland China. Exploring a possible mechanism that may expound the contingent effect of psychological safety is another purpose of our study. For this, we hope to make several contributions to both moral leadership and voice behavior by considering that employees' positive psychological safety is the key mechanism which makes moral leadership more or less effective.

\section{THEORY AND HYPOTHESES}

\subsection{Moral leadership and innovation performance}

Social exchange theory provides the theoretical support for our study. Employees under moral leadership are inclined to endow their leaders with extremely strong positive moral character, approve leaders' pursuit of collective goals, and try harder for the interests of the collective. When leaders show the quality of impartial and honest, followers are more likely to perceive them as intrinsically motivated and tend to identity the leader's values, being attracted to input constructive ideas and these creative ideas will in turn lead to higher innovation performance4. Apart from theoretical perspective, early researches also consider that moral leadership is positively correlated with employee's innovation performance 56. As Already Indicated, moral leadership is generally described as leaders' good behavior that shows outstanding moral quality and honest through disinterestedly actions and make themselves an example. As a return, employees are encouraged to demonstrate positive behaviors and improve their innovation performance to reciprocate. On the basis of frontal discussion, our first hypothesis is as follows:

Hypothesis 1: Moral leadership is positively correlated with innovation performance.

\subsection{The mediating effects of voice behavior}

In pursuit of organizational interests, moral leaders generally attract employees to adopt proactive behaviors to achieve higher innovation performance and then promote organizational development7. Voice represents a kind of promoting and challenging citizenship behavior8. In other words, voice challenges the unreasonable rules and tries to push forward aspiring organizational reform. So we propose voice behavior as the mechanism linking moral leadership and innovation performance in this study. This variable demonstrates proactive behavior aimed at creating beneficial conditions for further development. Examining voice behavior is helpful for us to understand how moral leaders do to encourage employees to put forward meaningful advice.

Although it intends to advance rather than merely criticize, voice behavior may bring potential risks to the proactive followers because it may challenge the authority of the leadership. Employees are less likely to take voice behavior if they feel that their opinions will be ignored or worry that they will be punished for conveying them.

Moral leadership is such a kind of leadership behavior, which can provide high moral standards, so it can effectively alleviate this situation. A leader of high morality shows self-discipline and selfless behaviors and plays a role model for individuals. This kind of leadership behavior will make employees break their obedience to formal agreements and encourages employees to intend to advance rather than merely criticize. In return, this increases employees' confidence in judging leaders and become courageous to voice their ideas, so as to put forward constructive comments.

We consider that moral leadership will not only promote voice behavior, but also lead to higher innovation performance in turn. Voice behavior plays a certain role in improving the functions of work unit and organizations9. In addition, the quality of decision-making will be enhanced when group members share divergent viewpoints 10 . These findings hold out the conjecture that employees will tend to have higher innovation performance when they put up more voice rather than less. Therefore, we think that voice behavior may be well stimulated by moral leaders to gain their innovation performance. Therefore, our second hypothesis is as follows:

Hypothesis 2: The positive relationship between moral leadership and innovation performance is mediated by voice behavior.

\subsection{The moderating role of psychological safety}

Moral leadership encourage employees to put up their thoughts, however, individuals may not have abundant confidence to carry out these proactive behaviors. This is because voice behavior may increase risk and criticism as a result of posing a divergent perspective. In this process, employees' choice of voice behavior will be moderated by their self-belief of working ability under specific situations. Psychological safety is the conception that captures accessible self-belief, it provides a psychologically secure context for followers 11 .

Psychological safety implies that individuals have faith in their image, position and career are not subject to 
the negative evaluation, therefore, they believe that their behavior will not be criticized when truthfully express their real opinions, namely the individuals can feel comfortable in interpersonal interaction and willing to make suggestions. This, to a large extent, alleviates employees' concerns about the potential risks and difficulties involved in voice behavior.

The innovation performance level of a follower may be related to his/her willingness to voice12. As shown in the previous, voice behaviors play a significant role in improving employees' innovation performance. However, the positive impact of voice behavior on innovation performance is not generated under all conditions, that is, the relationship is not a simple direct effect but moderated by some situational variables. Because of the buffering function, psychological safety has been conceptualized as a type of subjective situational variable that moderates the relationship between the two. We also consider that psychological safety is the moderator of the relationship between moral leadership and innovation performance such that the relationship is stronger when the psychological safety level is high.

To be specific, when employees have high level of psychological safety, they perceive to be able to freely express their views in the work will undertake less risk and thus are likely to put up constructive ideas to further enhance their innovation performance. As such, when seeking the chance to heighten innovation performance, they tend to consider voice behaviors because their high level of psychological safety will mitigate their fears and thus help unleash the potential of voice behaviors. In summary, we hold that high level of psychological safety helps employees to carry out voice behavior and to bring out innovation performance, thus strengthening the relationship between the two. By contrast, when employees' psychological safety level is low, they consider that they can't express their worries and concerns freely. They may still hold the assumption that there are more difficulties and heavier psychological stress when pose questions, even if they recognize the significant chance to promote innovation performance. In the face of the possibility to improve innovation performance, they tend to take the prevention-oriented method and pay more attention to the potential risks of voice behavior rather than the performance improvement they can obtain. As a result, they are less likely to promote their innovation performance by voice behavior. In general, we consider that low level of psychologically safe restrain voice behaviors, thus impairing the relationship between the two. On the whole, we proposed two moderating hypotheses as follows:

Hypothesis 3a: Psychological safety moderates the relation between moral leadership and voice behavior which is stronger when the level of psychological safety is low.

Hypothesis 3b: Psychological safety moderates the relationship between innovation performance and voice behavior, which is stronger when the level of psychological safety is high.

To sum up, we have hypothesized that there is a positive correlation between moral leadership and innovation performance, and voice behavior plays a mediating role. We further suggest that psychological safety plays a moderating role on the relationship between moral leadership and voice behavior, and the relationship is stronger with low rather than high psychological safety. Meanwhile, it also moderates the relationship between innovation performance and voice behavior, which is stronger with high rather than low psychological safety. Combining of all the above hypotheses, we considered that the positive indirect effect (via voice behavior) of moral leadership on innovation performance is moderated by psychological safety and when the psychological safety level of followers is low, the moderating effect become stronger. In particular, moral leaders' behaviors are more capable to meet the requirements of employees with lower psychological safety level, moral leadership should contribute more to the employees' innovation performance whose psychological safety level is low. By contrast, the relationship between moral leadership and innovation performance is undermined for employees with higher level of psychological safety, because they depend more on psychological safety than leaders' behavior to realize high level of innovation performance. Generally, our fourth hypothesis is as follows:

Hypothesis 4: The mediation of voice behavior make up the foundation of the entire moderating effect of psychological safety on the relationship between moral leadership and employees' innovation performance.

\section{METHOD}

\subsection{Sample and procedure}

Our sample is composed of 300 leader-subordinate paired data from four companies located in Mainland China. We divided the subordinates and their direct supervisors into two groups for questionnaire survey. We ensured the anonymity of everyone's answer in order to remove their doubts. In the end, 47 leaders and 224 followers responded.

In the supervisor data, 63 percent of the sampled supervisors were male, who averaged 47.3 years of age, and 64.4 percent had received a undergraduate or higher education. $71.3 \%$ of the followers were male and $64.4 \%$ had received a primary education. What we concluded from the sample was that the supervisors' education level is higher and their age and job tenure are senior as well.

\subsection{Measures}

For the sake of ensuring the questionnaires' validity and reliability, our study is based on the relatively mature scales and adjusts according to actual condition. Procedurally, the supervisors were required to evaluate their followers' innovation performance and voice behavior, while followers were supposed to privately evaluate their supervisors' moral leadership.

In this study, we used a 5-point Likert scale from 1 (strongly disagree) to 5 (strongly agree) to measure all main variables. We followed the standard translation and back-translation procedure to translate all English original measures into Chinese.

Moral leadership. We measured moral leadership 
using 9-item scale proposed by Cheng et al13. A sample item is "My supervisor treats us with fairness and selflessness". The $\alpha$ in this study was .87 .

Voice behavior. A 10-item scale used by Liang et al14 was used to measure Voice behavior. A sample item is "Raise suggestions to improve the unit's working procedure.". The $\alpha$ in this study was .96 .

Psychological safety. A 5-item scale used by Liang et al14 was used to measure Psychological safety. A sample item is "In my work unit, I can freely express my thoughts". The $\alpha$ in this study was .82 .

Innovation performance. We measured Innovation performance using 8-item scale proposed by Janssen et al15. A sample item is "He/She will searching out new working methods, techniques, or instruments". The $\alpha$ in this study was .96 .

Control variables. In order to avoid the confusion of interests, we controlled five individual demographic characteristics in the analysis, namely, gender, age, educational level, organizational tenure and income.

\section{Results}

\subsection{Confirmatory factor analysis}

We examine the distinctiveness of the proposed model by confirmatory factor analysis. The results of our four-factor baseline model (Moral leadership, Innovation performance, Psychological safety, and Innovation performance $)$ showed excellent fit $\left(\mathrm{x}^{2}=40.559, \mathrm{df}=344\right.$, $\mathrm{x}^{2} / \mathrm{df}=1.164 \quad \mathrm{RMSEA}=.017, \quad \mathrm{CFI}=.996, \quad \mathrm{GFI}=.092$, $\mathrm{TLI}=.995)$. The results supported the discriminant validity of our variables.

\subsection{Confirmatory factor analysis}

Descriptive statistics results are reported in Table I which also shows the correlations among all the variables. As can be seen from the table I, moral leadership is positively related to voice behavior $(\beta=.35, \mathrm{p}<.001)$, and Innovation performance $(\beta=.34, \quad p<.001)$. Voice behavior was positively related to Innovation performance $(\beta=.83$, $\mathrm{p}<.001)$ as well.

TABLE I. CORRELATIONS OF THE VARIABLES

\begin{tabular}{lllllll}
\hline Variable & M & SD & 1 & 2 & 3 & 4 \\
\hline $1 \mathrm{ML}$ & 3.68 & .77 & $(.87)$ & & & \\
$2 \mathrm{VB}$ & 3.21 & .74 & $.35^{* * *}$ & $(.96)$ & & \\
$3 \mathrm{PS}$ & 3.49 & .64 & $.42^{* * *}$ & $.20^{* *}$ & $(.82)$ & \\
$4 \mathrm{IP}$ & 3.22 & .80 & $.34^{* * *}$ & $.83^{* * *}$ & $.22^{* * *}$ & $(.96)$ \\
\hline
\end{tabular}

Note: ML(Moral leadership), VB (Voice behavior), PS (Psychological safety), IP (Innovation performance). $* \mathrm{p}<.05, * * \mathrm{p}<.01, * * * \mathrm{p}<.001$. (The same as in the following table.)

\subsection{Test of the Hypothesized Models}

\subsubsection{The main effect of moral leadership}

On the premise of controlling age, gender, education level, tenure and income level, we examined all the proposed relationships. According to the Model 6 in table II, moral leadership had a positive effect on innovation performance $(\mathrm{r}=.314, \mathrm{p}<.001)$. Thus, hypothesis 1 received support.

\subsubsection{The mediating role of voice behavior}

The first two conditions for examining a mediating relationship are that the independent variable (i.e. moral leadership) is related to the dependent variable (i.e. innovation performance), which is strongly supported by the analysis results of Hypothesis 1. In addition, moral leadership is related to voice behavior $(\mathrm{r}=.331, \mathrm{p}<.001)$ and voice behavior is also positively associated with innovation performance $(\mathrm{r}=.806, \mathrm{p}<.001)$. Voice behavior was significantly related to innovation performance $(\mathrm{r}=.797, \mathrm{p}<.001)$, while at this time, moral leadership was no longer significant. The above-mentioned results show that voice behavior plays a mediating role on the relationship between moral leadership and innovation performance. Therefore, Hypothesis 2 is supported.

\subsubsection{The moderating role of psychological safety}

We divided into two steps to verify the hypothesis $3 \mathrm{a}$ and 3b. To support Hypothesis 3a, from Table II we can see that, in the regression model, all the input variables in M1 are control variables, and then we input moral leadership in M2 and moderator variable (psychological safety) in M3.

As shown in M4 of Table II, we can see that the interactive effects of moral leadership and psychological safety on voice behavior $(\mathrm{r}=-.191, \mathrm{p}<.01)$ were significant. To support Hypothesis 3b, table II also showed a significant moderating effect of psychological safety between voice behavior and innovation performance. In particular, the $\beta$ for the interaction term interaction term (voice behavior * psychological safety) was significant for innovation performance $(\mathrm{r}=.087, \quad \mathrm{p}<.05)$. To materialization those interaction effects, the strength of the relationship between moral leadership and voice behavior was stronger when psychological safety is low $(\mathrm{r}=.473, \mathrm{p}<.01)$, the relationship between voice behavior and innovation performance was stronger when psychological safety is high $(r=.937, p<.001)$. Thus, Hypothesis $3 \mathrm{a}$ and $3 \mathrm{~b}$ is supported.

In Hypothesis 4, we further proposed that the indirect effect of moral leadership on employees' innovation performance through voice behavior, shows different strength in high and low levels of psychological safety. Hence, we used the approach of moderated path analysis to estimate the two sets of effects at the high and low levels of the psychological safety (i.e., moderating variable). The first-stage effect refers to the effect of moral leadership on voice behavior. The second-stage effect refers to the effect of voice behavior on innovation performance. The direct effect refers to the effect of moral leadership on innovation performance. And the overall indirect effect refers to the effect of moral leadership on innovation performance through psychological safety. The results indicate that the indirect relationship between moral leadership and innovation performance via voice behavior was significant 
$(b=.22, p<.01)$ under high level of psychological safety, and the indirect relationship between moral leadership and innovation performance via voice behavior was also significant $(b=33, p<.01)$ under low level of psychological safety. There is significant difference between the indirect relationships $(\Delta \mathrm{r}=-.12, \mathrm{p}<.05)$. Consequently, Hypothesis 4 is supported.

\section{Discussion}

The goal of our research was to examine the role that employees' positive psychological safety and voice behavior may play in the relationship between moral leadership and employees' performance. The results of our research reveals that moral leadership is positively associated with subordinates' voice behavior and therefore innovation performance, and to a great extent, among employees with low rather than high psychological safety level. Furthermore, psychological safety also moderates the relationship between voice behavior and innovation performance, which is much stronger among employees with high rather than low level of psychological safety. These researches are not only of theoretical significance, but also of practical significance.

\subsection{Theoretical significance}

First of all, we found that there is a positive link between moral leadership and employees' innovation performance, and the supervisors' moral leadership behaviors may affect the subordinates' innovation performance through voice behavior. Moral leadership intrinsically encourage followers to voice, which in turn leads to better innovation performance. Our research results suggest that, for understanding how moral leadership behaviors or organizational practices affects employees' innovation performance, it is beneficial for future researchers to consider various mechanisms when explaining the influence of these behaviors and practices.

Secondly, the contribution of our study lies in uncovering a positive association linking moral leadership to employees' voice behavior, which is consistent with other studies on the relationship between leadership behavior and employees' voice behavior to a certain extent.

The perceived morality of superiors acts as a significant role in shaping the effect of subordinates' voice behavior. Moral leadership behavior can contribute to subordinates developing a stronger acceptance to their superiors and can more actively explain the reactions of their superiors to their own views, suggesting to subordinates that there is no danger in giving advice and making suggestions, as superiors with morality would be supposed to be receptive to voice behavior and should not respond negatively. Hence, our studies consider that leadership behaviors and followers' psychological safety are conductive to followers' innovation performance in view of complementary congruity theory. Specifically, we hold that the relationship between moral leadership and voice behavior is stronger among employees whose psychological safety level is low.

Thirdly, this study is empirically testing the proactive behaviors (i.e., voice behavior) as a mediation mechanism of positive correlation between moral leadership and subordinates' innovation performance. Although one of the key assumptions of voice literature is that this behavior has positive performance effects, the relationship between voice behavior and innovation performance has received little attention from empirical research. Our research shows that an important advantage of moral leadership is that it can provide employees with an environment which they can speak freely and to put forward new ideas, thus improving their innovation performance. Especially, this study uncovers a mechanism through which moral leadership achieves complementarity with subordinates' requirements by demonstrating strict self-restraint, selfless behavior and acting as a personal role model, and then in turn results in their innovation performance.

\subsection{Management enlightenment}

Besides theoretical significance, our studies also have management enlightenment. The first thing that bears the brunt is that we have a new understanding of indigenous moral leadership behaviors studied in China. Although it is a leadership concept couched within the Chinese cultural tradition, it is positively related with subordinates' innovation performance. This study is helpful to managerial practitioners by pointing that moral leadership in line with the development of the modern society, and administrators who what to gain the trust of their subordinates and stimulate their suggestions would be more inclined to adopt moral leadership. Therefore, in the light of our results, supervisors are advised to adopt moral leadership and spend more effort on promoting subordinates with less positive psychological safety in order to motivate subordinates' voice behavior and thereby promote innovation performance.

Moreover, the findings of our research emphasize the practical value of voice behavior for innovation performance. So as to improve the quality of followers who are willing to put up different ideas, opinions and raise creative suggestions, then decision quality is enhanced. Therefore, the managers of organizations managers should think about diverse ways to motivate and support the voice behavior within the working groups, while leaders of the working groups should concentrates on cultivating supportive team voice climates. At the same time, they should try their best to encourage the progress of behavioral standards around responsibility.

Finally, ways for managers to help followers promote their innovation performing is to demonstrate nobel moral standards and exemplary moral conduct and create a positive psychological safety atmosphere.

In summary, by combining moral leadership and psychological safety, our study find the relationship between moral leadership and employees' voice behavior contingent on employees' psychological safety. Meanwhile, these results have also clearly proved that moral leadership can influence followers' innovation performances through a pivotal mechanism (e.g. voice behavior) and deepen our understanding on the complexities of moral leadership and on how it can be 
more availably implemented for employees' promoted voice behavior.

\subsection{Limitations}

First, this is a cross-sectional study, and we are unable to draw definite conclusions about causation. A second potential limitation of our study is about common method bias. Although we collected information about moral leadership from subordinates and subordinates' voice behavior and innovation performance from leaders, the data correlation between voice behavior and innovation performance may be relatively high because they come from the same source (i.e. leader). Therefore, we suggest that future research could benefit from a longitudinal design and data collection from multiple sources. At the same time, it is suggested that future researchers use objective performance or other-source rated innovation performance. Finally, leadership may vary across cultures. Based on this, the future research should study the moral leadership model in different cultures in order to gain more comprehensive research significance.

\section{CONCLUSION}

As one of the typical local leadership style in China, moral leadership has an important influence on contemporary managers in Chinese organizations. With this focus, we hope to make some significative contributions on both theoretical and empirical aspects to the development of business management under the background of Chinese traditional culture. This study develops and examines the theoretical relationship between moral leadership and employees' innovation performance, and discusses the mediating role of voice behavior in explaining the impact of moral leadership on followers' innovation performance. We also introduce psychological safety as a moderator variable in order to better explain the results. The results of this study emphasize the importance of moral leadership to employees' innovation performance. Future research may benefit from examining how organizations improve the moral competence of leaders and how moral competence differs from other types of competence in influencing employees' performance.

TABLE II. RESULTS OF REGRESSIONS

\begin{tabular}{|c|c|c|c|c|c|c|c|c|c|c|c|}
\hline \multirow{2}{*}{ Variable } & \multicolumn{4}{|c|}{ Voice behavior } & \multicolumn{7}{|c|}{ Innovation performance } \\
\hline & M1 & $M 2$ & M3 & M4 & M5 & M6 & M7 & M8 & M9 & $M 10$ & M11 \\
\hline Gender & -.011 & -.02 & -.013 & -.026 & -.036 & -.045 & -.029 & -.035 & -.047 & -.027 & -.024 \\
\hline Age & -.11 & -.092 & -.089 & -.075 & .021 & .038 & .112 & .043 & .056 & .116 & .109 \\
\hline Education & .134 & .086 & .090 & .124 & .137 & .091 & .022 & .097 & .130 & .031 & .031 \\
\hline Tenure & .051 & .071 & .069 & .077 & -.084 & -.065 & -.122 & -.067 & -.059 & -.121 & -.122 \\
\hline Income & .042 & -.003 & -.012 & -.023 & .069 & .026 & .028 & .014 & .003 & .021 & .026 \\
\hline ML & & $.331^{* * *}$ & $.305^{* * *}$ & $.340^{* * *}$ & & $.314^{* * *}$ & .048 & $.277^{* * *}$ & $.311^{* * *}$ & .040 & .051 \\
\hline PS & & & .063 & .073 & & & & .090 & .099 & .041 & .049 \\
\hline ML* PS & & & & $-.191^{* *}$ & & & & & $-.183^{* *}$ & -.030 & -.058 \\
\hline VB & & & & & & & $.806^{* * *}$ & & & $.797^{* * *}$ & $.792^{* * *}$ \\
\hline VB*PS & & & & & & & & & & & $.087^{*}$ \\
\hline$\Delta \mathrm{R}^{2}$ & .028 & $.102^{* * *}$ & .003 & $.033^{* *}$ & .044 & $.092^{* * *}$ & $.565^{* * *}$ & .006 & $.030^{* *}$ & $.530^{* * *}$ & $.007^{*}$ \\
\hline $\mathrm{F}$ & 1.271 & $5.401^{* * *}$ & $4.736^{* * *}$ & $5.362^{* * *}$ & 1.991 & $5.664^{* * *}$ & $71.997^{* * *}$ & $5.100^{* * *}$ & $5.593^{* * *}$ & $56.024^{* * *}$ & $51.83^{* * *}$ \\
\hline
\end{tabular}

\section{REFERENCES}

1. Manev I M, Elenkov D S. DS Elenkov, IM Manev 2005. Top management leadership and influence on innovation: The role of sociocultural context. Journal of Management, 31(3), 381-402[J]. Journal of Management, 2005, 31(3):381-402.

2. Osborn R N, Marion R. Contextual leadership, transformational leadership and the performance of international innovation seeking alliances[J]. Leadership Quarterly, 2009, 20(2):191-206.

3. West $\mathrm{M}$ A, Anderson $\mathrm{N}$ R. Innovation in top management teams[J]. Journal of Applied Psychology, 1996, 81(6):680-693.

4. Chan,S.C. Paternalistic leadership and employee voice: Does information sharing matter[J]. Human Relations, 2013, 67(6):667-693.
5. Aycan Z, Kanungo R N, Sinha J B P. Organizational Culture and Human Resource Management PracticesThe Model of Culture Fit[J]. Journal of Cross-Cultural Psychology, 1999, 30(4):501-526.

6. Chen X P, Eberly M B, Chiang T J, et al. Affective Trust in Chinese Leaders: Linking Paternalistic Leadership to Employee Performance[J]. Journal of Management, 2011, 40(3):págs. 796-819.

7. Walumbwa F O, Morrison E W, Christensen A L. Ethical leadership and group in-role performance: The mediating roles of group conscientiousness and group voice[J]. Leadership Quarterly, 2012, 23(5):953-964.

8. Dyne L V, Cummings L L, Parks J M. Extra-role behaviours: In pursuit of construct and definitional clarity (a bridge over muddied waters) [J]. Research in Organizational Behavior, 1995, 17:215-285.

9. Morrison E W, Wheeler-Smith S L, Kamdar D. 
Speaking up in groups: a cross-level study of group voice climate and voice.[J]. Journal of Applied Psychology, 2011, 96(1):183.

10. Nemeth C J, Connell J B, Rogers J D, et al. Improving Decision Making by Means of Dissent[J]. 2001, 31(1):48-58.

11. Edmondson A. Psychological Safety and Learning Behavior in Work Teams[J]. Administrative ence Quarterly, 1999, 44(2):350-383.

12. Ashford S J, Rothbard N P, Piderit S K, et al. Out on a Limb: The Role of Context Impression Management in Selling Gender-Equity Issues[J]. Administrative science Quarterly, 1998, 43(1):23-57.
13. Cheng Borshiuan, Chou Lifang, Farh Jiinglih. Paternalisticleadership scale:construction and measurement of triad model (in Chinese). Indigenous Psychological Societies, 2000, 13:127-180.

14. Liang, J., Farh, C. I., \& Farh, J. L. (2012). Psychological antecedents of promotive and prohibitive voice: A two-wave examination. Academy of Management Journal, 55(1), 71-92.

15. Janssen O, Yperen N W V. Employees' Goal Orientations, the Quality of Leader-Member Exchange, and the Outcomes of Job Performance and Job Satisfaction[J]. Academy of Management Journal, 2004, 47(3):368-384. 